\title{
Los diálogos transdisciplinarios: Una forma de articular la Universidad y las organizaciones campesinas
}

\section{Dialogues transdisciplinary: A way of articulate University and rural organizations}

\author{
Juan Ignacio Alfaro-Mardones, Carmen Fernández-Hernández, Manuel González-García \\ FAREM-Matagalpa, UNAN-Managua, altarrib@ibw.com.ni, carmenferher@yahoo.com,mjesus14@yahoo.com
}

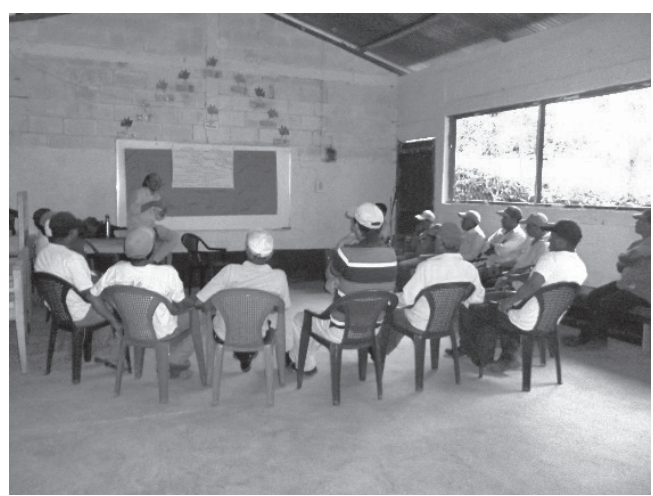

\section{RESUMEN}

El desarrollo de los territorios y sujetos rurales necesita de nuevas formas de abordar la compresión de sus problemáticas. La universidad y la Unión de campesinos organizados de San Dionisio (UCOSD) llevan desde el 2013 un proceso de diálogo transdisciplinario para generar estrategias de desarrollo económico, humano y de adaptación al cambio climático desde las familias de trece comunidades rurales. A partir de la matriz de necesidades y satisfactores se lograron detectar las principales carencias y potencialidades desde cada comunidad para así identificar los satisfactores sinérgicos y las patologías principales. La destrucción del tejido comunitario, por la desconfianza y la falta de coherencia personal del liderazgo. Esta información se analizó a la luz del plan estratégico de la organización y se obtuvieron unos aprendizajes y líneas de acción: La necesidad de afrontar los conflictos que cada persona arrastra para poder fortalecer la organización, el papel que la asociatividad juega en esa sanación personal, la necesidad de diálogo entre la memoria organizativa con las expectativas del relevo generacional. Este diálogo continúa y aspira a clarificar el futuro de la ruralidad en el territorio de San Dionisio y Matagalpa.

Palabras clave: Transdisciplinariedad, investigación acción participativa, necesidades, satisfactores.

\section{ABSTRACT}

The development of rural territories and subjects need for new approaches to understanding their problems. The university andthe Union of peasants organized in San Dionisio (UCOSD lead from the 2013 transdisciplinary dialogue process to generate strategies for economic, human development and adaptation to climate change from the families of thirteen rural communities. From the array of needs and satisfactions were able to identify major gaps and potentials from each community in order to identify the synergic satisfiers and major diseases. The destruction of the social relation, by mistrust and lack of personal integrity of leadership. This information is analyzed in light of the strategic plan of the organization and a learning and action lines were obtained: the need to heal the conflicts that each person drags to strengthen the organization, the role the association plays in the healing, the need for dialogue between organizational memory generational expectations. This dialogue continues and aims to clarify the future of rurality in the territory of San Dionisio and Matagalpa

Key words: Transdisciplinarity, participatory action research, needs, satisfactions 
$\mathrm{L}$ a Universidad Nacional Autónoma de Nicaragua, mediante su Facultad Regional Multidisciplinaria de Matagalpa (UNAN-FAREM-Matagalpa) ha establecido alianzas con la Unión de Campesinos Organizados de San Dionisio (UCOSD) a partir de una serie de acciones concretas en diferentes marcos de actuación: tesis de grado y prácticas profesionales desde las carreras de ingeniería agronómica y economía; tesis de maestría en el programa de Maestría en desarrollo rural territorial sustentable; una propuesta de investigación, en el marco del proyecto de la Sociedad Rural, Economía y Recursos Naturales, Integrando competencias en el Desarrollo Rural" (SERIDAR) (Sociedad rural, economía y recursos naturales. Integrando competencias en el desarrollo rural. , 2013) y actualmente tres tesis en el programa de Doctorado en Desarrollo rural territorial sustentable que tratan de articular las prácticas y las tesis de investigación de pregrado de la carrera de economía con este programa de postgrado. De este vínculo, surgió la demanda de la organización para que les acompañáramos, mediante un proceso participativo y transdisciplinario, en el análisis de la realidad socioeconómica y productiva de las familias y comunidades, que permitiera generar un conjunto de propuestas de desarrollo, a ser implementadas por la organización o gestionadas ante otros actores locales.

¿Cómo está influyendo la asociatividad entre campesinos para los procesos de sanación personal en las familias rurales? ¿Quiénes son los agricultores del futuro en la subcuenca del Río Cálico? ¿Cuál es la mochila (PNUD, 2011) que están trasladando los campesinos a sus hijos e hijas? Una vez identificadas estas primeras preguntas vinculadas al desarrollo interno de las personas asociadas a esta organización campesina, se comenzó un proceso de reflexión comunitaria realizado en el año 2013 en el marco del proyecto SERIDAR. Desde enero del 2014 hasta junio 2014 se acompañó un proceso de reflexión comunitaria, que permitió posteriormente cruzar las necesidades recogidas en el plan organizativo institucional(UCOSD, 2011) de la UCOSD con las carencias y las potencialidades que desde cada una de las comunidades se han planteado. Esta información fue analizada conjuntamente con el consejo directivo de la UCOSD y el equipo transdisciplinario de la FAREM Matagalpa para definir las acciones de investigación que determinaría la continuidad de este diálogo entre la academia y el campesinado en el territorio de San Dionisio. Este artículo analiza la construcción de este diálogo entre la universidad y las familias productoras para generar un nuevo conocimiento para afrontar los retos del cambio climático, la seguridad alimentaria y del desarrollo humano.

\section{MATERIALES Y MÉTODOS.}

La necesidad de la transdisciplinariedad. Las organizaciones humanas, en el contexto rural, son capaces de generar relaciones sinérgicas que satisfacen al mismo tiempo diferentes necesidades del desarrollo. Las Universidades han pri- vilegiado el lado externo del desarrollo, pues los andamiajes teóricos y metodológicos del empirismo sensitivo se adaptaban mejor a estas superficies del desarrollo, pero a partir de un diálogo transdisciplinario con los y las protagonistas del desarrollo se podrían identificar significativas interioridades y nos permitirían tener una mirada más integral y sistémica de estos procesos que nos permitan comprenderla mejor. "El problema no está en demostrar con pruebas científicas que el desarrollo tiene problemas, como la industrialización, la sobreexplotación de los recursos, el cambio climático, la desnutrición, analfabetismo o cualquier otra cosa. Las pruebas de que es así son simples y abrumadoras. Cualquiera puede entender los datos empíricos (cuantitativo), pero a la mayoría de la gente no les importa (cualitativo). El principal problema es la falta de comprensión mutua y de entendimiento de estos problemas desde una conciencia mundial. El problema real no es exterior, sino interior"(Wilber, 2005). El diálogo transdisciplinario permite la complementariedad entre las ciencias naturales que se ocupan de los aspectos tangibles del desarrollo rural y los aspectos intangibles e inmateriales presentes y cada vez más importantes para poder hablar de procesos de desarrollo. En este diálogo, Saúl Úbeda, fundador de la UCOSD y actualmente coordinador contratado por el consejo directivo, nos recuerda desde su experiencia a la academia del peligro de la fragmentación. "Mis mejores momentos eran cuando todos los productores éramos parte de la asociación. La institucionalidad (burocratización) nos dividió y la profesionalización nos fragmentó" (Úbeda, 2014).

La investigación acción participativa como marco metodológico para el desarrollo. La Unión de Campesinos Organizados de San Dionisio (UCOSD), es un sujeto colectivo organizado, que ha buscado el desarrollo de sus asociados. A partir de un proceso de reflexión colectiva, iniciado hace aproximadamente hace treinta años, esta organización se ha ido transformando como respuesta a las necesidades internas de sus asociados y a los estímulos externos del contexto sociopolítico y la dinámica tecno económica. La universidad está desarrollando un dialogo con esta institución y sus participantes, para contrastar sus conocimientos adquiridos en este tiempo, con el saber teórico acumulado por las investigaciones de los docentes involucrados en este programa de investigación. Desde el 2013 hasta el 2015 se ha mantenido una relación de diálogo permanente donde tanto profesores, como estudiantes hemos participado y facilitado asambleas comunitarias y grupos focales. Escuchar y aprender de esta experiencia y al mismo tiempo dialogar con el resto de dimensiones externas de su desarrollo, en un proceso transdisciplinar, que apunte a generar esos cambios sociales. Un posicionamiento metodológico con la Investigación acción participativa (IAP), "procesos de investigación en un camino en "espiral" según palabras del educador australiano Stephen 
Kemmis (1989) encontró en esta modalidad de investigación el fundamento de una ciencia social crítica bien demarcada de las corrientes positivistas y hermenéuticas" (Ortiz \& Borjas, 2008). El proceso de problematización ha sido a partir de triangular y contrastar el plan estratégico institucional con las potencialidades y carencias manifestadas en las asambleas comunitarias desde las familias participantes. Este proceso de problematización dialogada se realizó a partir del análisis de toda la información de cada una de las comunidades y su contrastación con las líneas del plan estratégico comunitario. Esto ha dado lugar a una operacionalizacion consensuada de los aspectos que debemos abordar con la investigación acción desde cada una de las familias y parcelas.

La matriz de necesidades y satisfactores de Max-Neef, Elizalde y Hopenhayn como herramienta facilitadora de diálogos transdisciplinarios. "Lo que se ha sugerido en esta reflexión es que: a) cualquier necesidad humana fundamental no satisfecha de manera adecuada produce una patología; b) hasta el momento, se han desarrollado tratamientos para combatir patologías individuales o de pequeños grupos; c) hoy en día, nos vemos enfrentados a una cantidad de patologías colectivas que aumentan de manera alarmante, para las cuales los tratamientos aplicados han resultado ineficaces; d) para una mejor comprensión de estas patologías colectivas es preciso establecer las necesarias transdisciplinariedades " (Max-Neef, Elizalde, \& Hopenhayn, 1986). Esa mirada interior tiene que ser transdisciplinaria, pues debe de tratarse de una mirada dialogada, que nos permita integrar disciplinas para una mayor comprensión de la realidad."La sistematización propuesta es aplicable para fines de diagnóstico, planificación y evaluación. La matriz de necesidades y satisfactores puede servir, en primera instancia, de ejercicio participativo de auto-diagnóstico de grupos insertos en el espacio local. A través de un proceso de dialogo iterativo preferentemente con la presencia de un promotor que haga las veces de elemento catalizador- el grupo puede ir identificando sus características actuales en la medida en que sus integrantes vayan llenando los respectivos casilleros."(Max-Neef, Elizalde, \& HopenHopenhayn, 1986) hayn, 1986). El equipo investigador de la UNAN apoyados por los estudiantes generaron este diálogo dentro de las trece comunidades donde viven los productores y productoras asociadas a la organización. En este diálogo se profundizó en cada una de las necesidades desde sus diferentes categorías axiológicas y existenciales. "El diálogo puede ser el camino para el cambio.” (Karlsen \& Larrea, 2015)

En las trece comunidades, las personas asociadas a la UCOSD pudieron mirarse en este espejo y mirar así los satisfactores construidos desde las familias, desde la comunidad y la relación que tiene la organización campesina con estos satisfactores. Analizar lo más negativo y lo más positivo (satisfactores con menor y mayor sinergia) y de esta forma tomar conciencia y reflexionar sobre sus carencias y potencialidades.

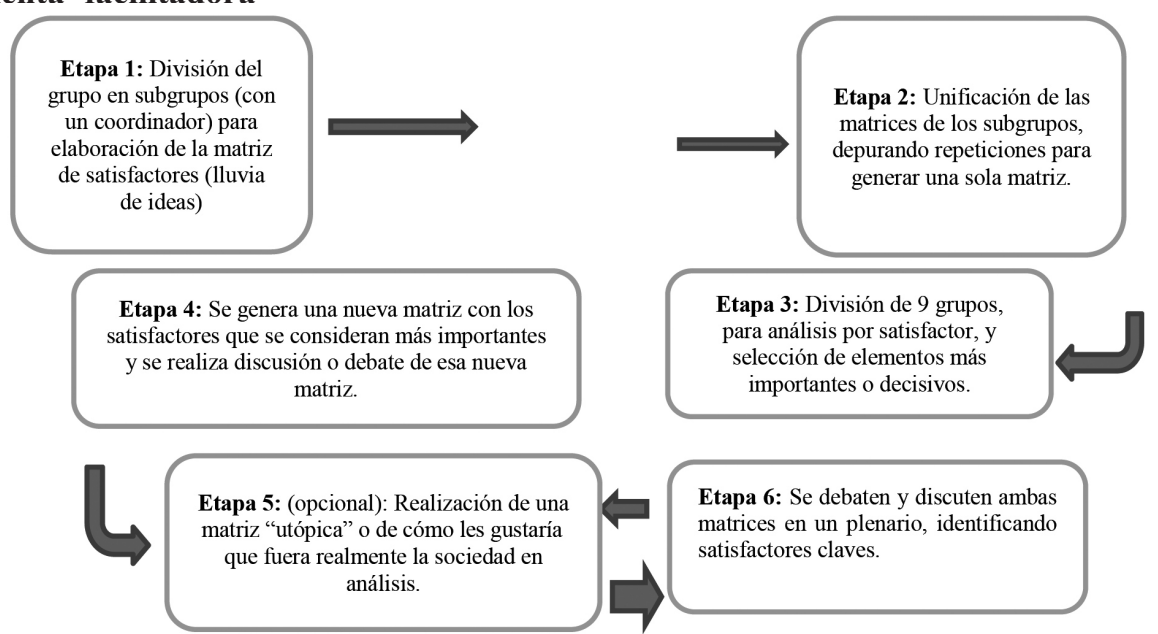

Figura 1. Etapas del proceso de aplicación metodológica de la matriz, propuestas por MaxNeef, Elizalde y Hopenhayn.Fuente: Elaboración propia a partir de (Max-Neef, Elizalde, \&



Figura 2. Metodología de aplicación de la matriz de DEH en las comunidades rurales.Fuente: Elaboración propia a partir de (Max-Neef, Elizalde, \& Hopenhayn, 1986).

Todo el proceso nos permitió operacionalizar la estrategia de investigación y de acción para continuar este proceso de transformación productiva, institucional y sobre todo humana. 


\section{RESULTADOS Y DISCUSIÓN}

Una vez analizada de forma transversal e identificadas los elementos comunes y diferenciados que podrían ser explicados por necesidad, pudimos cruzar una serie de información generada por este diagnóstico transdisciplinario comunitario con las líneas de acción y estrategias que la organización tenían planteadas en su plan estratégico. De esta triangulación pudimos tomar conciencia de las potencialidades y carencias que cada comunidad presenta frente a los retos planteados por la institución en su plan estratégico.

Aprendizajes integradores. En una primera etapa desde el análisis del equipo de facilitadores comunitarios de la UCOSD y el equipo investigador de la UNAN se llegaron a consensuar las siguientes carencias y potencialidades transversales para las comunidades donde tiene presencia la UCOSD: carencias de liderazgo, participación de la mujer, mala comunicación, desconfianza y resentimientos, y problemas de entendimiento.

Las potenciales sinérgicas en las comunidades son identidad rural y comunitaria, experiencia organizativa, memoria organizacional, recursos productivos y producción, espíritu de cambio y nuevos socias. A estos se suman el potencial ecológico, las alianzas con otras organizaciones y el potencial de los jóvenes.

Las patologías sinérgicas detectadas por el equipo investigador en las comunidades se resumen de la siguiente forma. Desaliento organizacional (sin aprendizajes de los errores y proyectos de la UCOSD), fatiga del liderazgo comunitario y centralización gerencial y la falta de conexión entre la parcela, la transformación productiva y el destino de la producción.

En una segunda etapa donde se analizan y dialogan con los diferentes equipos de investigación y las personas que integran el consejo directivo se llegó a las siguientes conclusiones; necesidad de ampliar la reflexión comunitaria sobre la visión gremial, seguridad del campesino, cambio climático, entendimiento en general; y necesidad de trabajos de reflexión técnicos, empresariales, productivos y ambientales, no bajo la capacitación tradicional, sino tallares prácticos a partir de las experiencias, de cómo se hacían las cosas y como se pueden cambiar y transformar desde los actores.

A partir de estas dos conclusiones tanto la universidad como la organización campesina tratan de delimitar sus capacidades y voluntades para poder asumir compromisos y responsabilidades en las nuevas etapas que alianza y colaboración de la cual esta investigación doctoral es parte.

Recomendaciones organizacionales. El consejo directivo de la UCOSD pide una serie de recomendaciones hacia la organización que nazcan del análisis transdisciplinar de esta primera fase de la investigación. Las mismas se resumen de la siguiente forma:
- Rotación en las responsabilidades

- Trabajo en la comunicación organizacional

- Forma pedagógica y didáctica de afrontar el entendimiento

- Análisis de aprendizajes sobre la innovación práctica y empírica realizada

- Trabajar el relevo generacional

- Ordenar la organización a lo interno. Descentralización, rotación.

- Apoyo organizacional a la innovación.

- Reflexión permanente y análisis sobre los programas desde el punto de vista de la equidad (comunitaria, intergeneracional, de género, etc...)

- Redimensionar el papel de la mujer

- Incentivar la asistencia en la reflexión y trabajo gremial, a partir de acceso a programas. Fuente: Equipo investigador.

Consolidado aprendizajes. Finalmente en cuatro sesiones de diálogo transdisciplinar entre los docentes, grupos de estudiantes y consejo directivo de la UCOSD se llegaron a los siguientes aprendizajes de esta primera fase.

- Necesidad del dialogo transdisciplinar

- Nueva pedagogía

- (empirismo sensitivo)

- Base ética del desarollo

- Asociatividad como vehículo de sanación

- Institucionalización versus organización comunitaria.

- Trabajar la memoria como un método de reflexión comunitaria y familiar. Fuente: Equipo investigador.

\section{CONCLUSIONES}

Los aprendizajes a enfrentar son las patologías y carencias sinérgicas que tienen paralizada la organización campesina. Dolores profundos por una falta de coherencia en el liderazgo, desconfianzas comunitarias y la necesidad de hacer las cosas diferentes. Sobre todo la necesidad de continuar este diálogo, usar didácticas más sensitivas, no perder la reflexión comunitaria aunque se necesite institucionalizar un movimiento social, el uso de la memoria como método de sanación personal y de diálogo generacional y saber que las alternativas pasan por el camino interior de la ética intersubjetiva y de la conciencia personal. En Nicaragua los procesos educativos que se proponían por parte de los movimientos campesinos se vieron truncados por la toma del poder con la revolución sandinista. El ideario de Sandino, la propuesta de Carlos Fonseca eran las transformaciones personales para cambiar Nicaragua. Este proceso interrumpido por el ejercicio del poder, causó mucho daño. Una represión del desarrollo humano por un desarrollo material. ¿Cómo afrontará estos procesos de sanación personal? Si logro abrir el alma, vivir los duelos de las ilusiones, sueños y conectarme así al resto del mundo. 
Todo diálogo siempre nos deja más interrogantes que respuestas. ¿Cómo responsabilizarme de un proceso de desarrollo, sino puedo responsabilizarme de mis propias contradicciones? ¿Cómo puede la universidad obviar sus contradicciones institucionales en un diálogo con las contradicciones campesinas? ¿Cómo dialogar desde mis propias contradicciones con las contradicciones de todas estas personas con las que estamos dialogando? Si las organizaciones tienen los mismos problemas básicos con la Universidad, desconfianzas comunitarias, líderes con su propia historia irresuelta, maquillaje de los dolores más profundos, vidas simuladas y apuestas por la superficialidad en las relaciones personales y abrazo al consumo como sustituto de la esencia existencial. ¿Cómo podemos apoyarnos con este diálogo? ¿Qué nuevo conocimiento mestizo e híbrido nacerá de estos diálogos? ¿Querrán esta nuevas generaciones escuchar la memoria de los fundadores de la organización? ¿Servirá escuchar esa memoria para que se encuentren con la parte de su identidad campesina? ¿Serán estos jóvenes los campesinos del futuro? ¿Estaré dialogando con los últimos campesinos en este territorio de este siglo XXI?

\section{REFERENCIAS BIBLIOGRÁFICAS.}

Karlsen, J; Larrea, M. (2015). Desarrollo territorial e investigación acción. Donostia - San Sebastian: Orkestra - Instituto Vasco de Competitividad.

Max-Neef, M; Elizalde, A; Hopenhayn, M. 1986. Desarrollo a escala humana: una opción para el futuro.Santiago de Chile: Centro de alternativas de desarrollo.

Ortiz, M; Borjas, B. 2008. La investigación acción participativa: aporte de Fals Borda a la educación popular. Espacio abierto. 17(4):615-625.

PNUD. (Programa de las Naciones Unidas). 2011. Informe Nacional sobre Desarrollo Humana 2011: Las juventudes construyendo Nicaragua 2011. Managua.

SERIDAR (Sociedad Rural, Economía y Recursos Naturales. Integrando competencias en el desarrollo rural). 2013. Proyecto de actualización de los enfoques y estrategias de desarrollo de la UCOSD. Matagalpa: SERIDAR - UNAN FAREM Matagalpa.

Úbeda, S. 2014. La UCOSD como actor social. San Dionisio.

UCOSD (Unión de Campesinos Organizados de San Dionicio). 2011. Plan Estratégico 2012-2016. Susulí: Programa de apoyo al fortalecimiento de la Unión de Campesinos Organizados de San Dionisio

Wilber, K. (2005). Sexo, ecología y espiritualidad. Madrid: GAIA ediciones. 